\title{
Coronal STIR sequence, a simple adjustment to routine MRI protocol for extra-spinal sciatica and sciatica-like symptoms
}

\author{
Rania Zeitoun * (1) and Mohammed Saleh Ali Mohieddin
}

\begin{abstract}
Background: The value of adding coronal STIR images to MR imaging of sciatica aiming to detect extra-spinal abnormalities.

Results: Additional coronal STIR images detected extra-spinal abnormalities in 20\% of the patients, thereby downgraded the normal studies from 21 to 13\%. The extra-spinal abnormalities included bone abnormalities (36.4\%), soft tissue abnormalities (4.5\%), neurological abnormalities (2.3\%), gynecological abnormalities (50\%), and miscellaneous (6.8\%). In 6.9\% of patients, the extra-spinal abnormalities explained the patients' pain and influenced their management. Extra-spinal causes of pain significantly correlated to positive trauma and neoplasm history, normal routine protocol images, and absent nerve root impingement. Extra-spinal abnormalities were more prevalent in age groups (20-39years).

Conclusion: Coronal STIR images (field of view: mid abdomen to the lesser trochanters) identify extra-spinal abnormalities that maybe overlooked on routine MRI protocol. It is of additional value in young adults, trauma, neoplasm, and negative routine images.
\end{abstract}

Keywords: Sciatica, Extra-spinal sciatica, Coronal, STIR

\section{Background}

Sciatica is defined as low back pain that radiates to the legs, with or without neurologic deficiency. Patients may present with non-radiating low back pain, buttock pain, thigh or leg pain, and sensory/motor deficits, which are referred to as sciatica-like symptoms [1]. In most cases, sciatica or sciatica-like symptoms is caused by lumbar nerve roots compression secondary to discogenic disease. However, in some cases, such symptoms may result from nerve compression in an extra spinal location where pathologies involving the lumbosacral plexus or sciatic nerve at the pelvis, gluteal region, and upper thigh are suggested to be the causative factor. Such conditions have been reported in literature as "extra spinal sciatica" [2, 3].

\footnotetext{
* Correspondence: raniazeitoun@gmail.com; rania.zeitoun@kasralainy.edu.eg Department of Diagnostic and Interventional Radiology, Kasr Al-Ainy Faculty of Medicine, Cairo University, Cairo, Egypt
}

Variable etiologies can be behind extra spinal sciatica: traumatic, infective, neoplastic, vascular, and gynecological. All of which might be easily overlooked on routine magnetic resonance imaging (MRI) protocol. Although MRI is the modality of choice for investigating sciatica, however its routine protocol, constituted by sagittal views of the lumbar spine and axial views along the disc planes, does not allow detection of extra spinal causes of sciatica or sciatica-like symptoms $[3,4]$.

Short TI inversion recovery (STIR) sequence is one of the inversion recovery (IR) pulse sequences and is the most commonly used. With STIR sequences, all tissues of short T1 relaxation times, including fat, are suppressed, whereas tissue with high water content, including most pathologic lesions, are accentuated, yielding a bright signal on a dark background of nullified short-T1 tissue [5]. Adding a STIR sequence in coronal view to cover the bony pelvic girdle and surrounding soft tissues, 
including the lumbosacral plexus and branches, is thought to be useful in detection of extra-spinal etiologies of sciatica and sciatica-like symptoms that may be missed on routine imaging protocol $[4,6,7]$.

The purpose of this study is to determine the value of adding a coronal STIR sequence to the routine MRI protocol in patients presenting with sciatica, aiming to detect overlooked extra spinal abnormalities and avoid delayed or mistreatment.

\section{Methods}

\section{Patients}

The present study was conducted in the authors' institute. We designed a prospective study which included patients presented with sciatica or sciatica-like symptoms and referred to perform lumbosacral MRI examination at our department. The study extended from October 2016 to April 2017. We excluded patients who had undergone a lumbar spine surgery or who have a documented recent spine fracture. A total number of 217 patients (84 males and 133 females, age range 1770 years and mean age 42.5 years) were included in our study. We interviewed the patients to record relevant data: pain analysis (true sciatica $(n=158)$, low back pain without radiculopathy $(n=41)$ and buttock pain $(n=$ $18)$ ), history of trauma $(n=5)$, neoplasm $(n=3)$, systemic disease, or medications.

\section{Magnetic resonance (MR) scanner}

All lumbosacral MR examinations were performed on a 1.5 Tesla MRI scanner (Achieva, Philips Medical Systems, The Netherlands). The patient lays supine, head first. The examinations are done using a dedicated spine coil. Cushions are used for immobilization and extra comfort for legs.

\section{Lumbosacral MRI protocol Routine MRI protocol}

The protocol consisted of sagittal T1W (slice thickness, $4 \mathrm{~mm}$; field of view (FOV), $280 \mathrm{~mm}$; matrix, $307 \times 384$; repetition time (TR)/echo time (TE), 400/8 and scan duration, $2.24 \mathrm{~min}$ ); sagittal T2W (slice thickness, $4 \mathrm{~mm}$; FOV, $280 \mathrm{~mm}$; matrix, $280 \times 384$; TR/TE, 3000/120 and scan duration, $1.6 \mathrm{~min}$ ); and axial T2W (slice thickness,

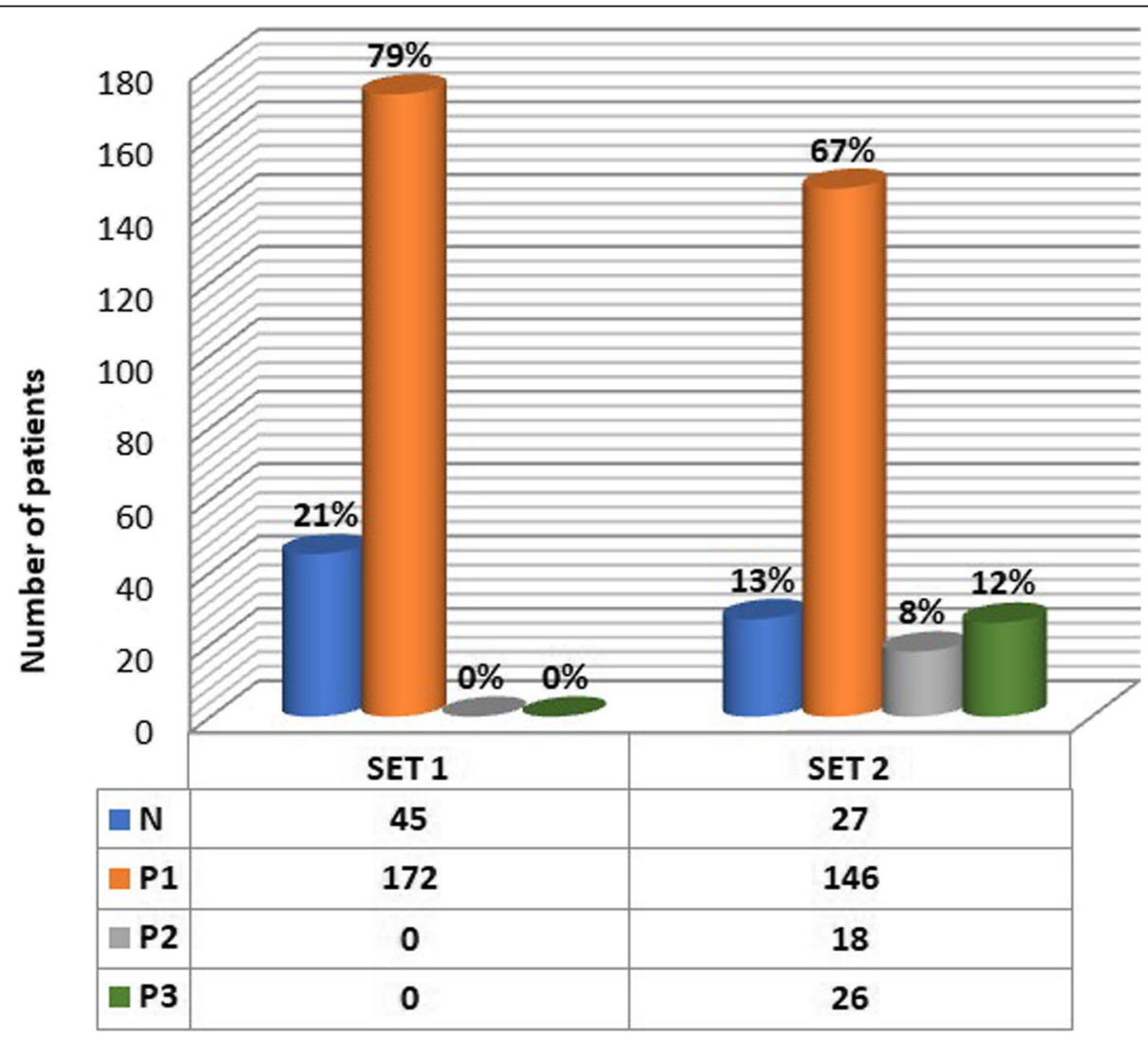

Fig. 1 A graph presentation of MRI findings as reported using set 1 (routine MR protocol images) and set 2 (routine protocol MR images and additional coronal STIR images). N normal MR images of the lumbar spine, P1 positive spinal abnormalities, P2 positive extra-spinal abnormalities and P3 spinal and extra-spinal abnormalities. The percentage of normal studies (blue column) has been downgraded from 21\% on set 1 to 13\% on set 2, on the expense of increase in the number of extra-spinal abnormalities (gray and green columns) detected after adding coronal STIR images 
$4 \mathrm{~mm}$; FOV, $230 \mathrm{~mm}$; matrix, $256 \times 256$; TR/TE, 3000 / 120 and scan duration $3 \mathrm{~min}$ ). Axial images were performed at selected levels chosen from the sagittal sequences, angled through the intervertebral discs.

\section{Additional coronal STIR sequence}

A coronal STIR sequence (slice thickness, $6 \mathrm{~mm}$; FOV, $500 \mathrm{~mm}$; matrix, $269 \times 384$; TR/TE, $2500 / 40$; inversion time (TI), 150 and scan duration, $3.20 \mathrm{~min}$ ) covering the paraspinal and pelvic areas was performed, ranging from the mid abdominal cavity to the lesser trochanters of the femur.

\section{Images interpretation and analysis of findings}

All images were transferred to a workstation using the Digital Imaging and Communications in Medicine (DICOM) format. The MR images were interpreted by two experienced musculoskeletal radiologists (11 and 25 years of experience). For each patient, we evaluated the routine MR protocol images: sagittal T1, sagittal T2, and axial T2 image (will further be referred to as set 1) and the routine protocol images in addition to coronal STIR images (will further be referred to as set 2). Set 1 images were categorized into $(\mathrm{N})$ normal MR images of the lumbar spine or (P1) positive spinal abnormalities, while set 2 images were categorized into $(\mathrm{N})$ normal MR images of the lumbar spine, (P1) positive spinal abnormalities, (P2) positive extra-spinal abnormalities, and (P3) spinal and extra-spinal abnormalities. The extra-spinal abnormalities which were recorded on set 2 images were further studied to select the abnormalities which the authors are confident that can explain the patient's pain or influence the management plan.

\section{Statistical analysis}

Statistical analyses were performed using the statistical package SPSS (Statistical Package for the Social Sciences) version 24. The extra-spinal abnormalities that were believed to explain the patient's pain or influence the management plan were correlated with age, sex, history of trauma, history of neoplasia, associated spinal abnormality, and associated spinal abnormality with nerve root impingement using chi-square test. Exact test was used instead when the expected frequency is less than 5 . $P$ values less than 0.05 were considered as statistically significant.

\section{Results}

The results of categorization of MR images findings are plotted in Fig. 1. Interpreting set 1 resulted in 45 normal patients (21\%); on the other hand, set 2 showed only 27 patients (13\%) as normal. On set 2, we could record extra-spinal abnormalities through interpreting the additional coronal STIR images in 44 patients $(20 \%)$, which were not visible on standalone routine protocol images (set 1). All the recorded extra-spinal abnormalities are listed in Table 1 including bone abnormalities $(n=16$; $36.4 \%)$, soft tissue abnormalities $(n=2,4.5 \%)$, neurological abnormalities $(n=1,2.3 \%)$, gynecological abnormalities $(n=22,50 \%)$, and miscellaneous $(n=3,6.8 \%)$. We selected 15 patients (6.9\%) who had extra-spinal abnormalities that were believed to explain patients' pain and influence their management plan (Table 2). These included bilateral sacroiliitis $(n=4)$ (Fig. 2), stress fracture of the sacral ala $(n=2)$ (Fig. 3), stress fracture of the proximal femur $(n=2)$, iliac bone metastases $(n=2)$ (Fig. 4), occult pelvic fracture $(n=1)$, occult intertrochanteric fracture $(n=1)$ (Fig. 5), transient osteoporosis of the hip (TOH) $(n=1)$, Tarlov cysts $(n=1)$ (Fig. 6), and gluteal muscle tendinopathy $(n=1)$.

Identification of these extra-spinal abnormalities was the primary focus of the additional STIR sequence. Correlation of the presence of extra-spinal abnormalities with age of patients proved significant in the age subgroup between 20 and 29 years old ( $P$ value $=0.002$ ). It was not significantly affected by patients' sex $(P$ value $=$ 0.228 ); conversely, it was significantly affected by positive history of neoplasia and trauma $(P$ value $=0.013$ and 0.04 respectively) (Table 3 ). It was significantly

Table 1 Extra-spinal abnormalities detected on coronal STIR images

\begin{tabular}{ll}
\hline Extra-spinal abnormality & No. of patients \\
\hline Bony abnormalities & $16(36.4 \%)$ \\
Bilateral sacroilitis & 4 \\
Stress fracture of proximal femur & 2 \\
Stress fracture of sacral ala & 2 \\
Iliac wing metastasis & 2 \\
Transient osteoporosis of the hip & 1 \\
Occult pelvic fracture & 1 \\
Occult intertrochanteric fracture & 1 \\
Simple bone cyst & 3 \\
Soft tissue abnormalities & $2(4.5 \%)$ \\
Gluteal muscular tendinopathy & 1 \\
Gluteal granulomas of IM injection & 1 \\
Neurological & \\
Tarlov cysts & $1(2.3 \%)$ \\
Gynecological abnormalities & $22(50 \%)$ \\
Ovarian cysts & 20 \\
Uterine fibroids & 2 \\
Miscellaneous & $3(6.8 \%)$ \\
Ectopic pelvic kidney & 1 \\
Enlarged prostate & 2 \\
Total & 44 \\
\hline
\end{tabular}


Table 2 Extra-spinal abnormalities detected on coronal STIR images, explain the patients' pain and influence their management

\begin{tabular}{|c|c|c|c|c|c|c|}
\hline \multirow{2}{*}{$\begin{array}{l}\text { Coronal STIR images } \\
\text { Extra-spinal abnormality }\end{array}$} & \multirow[t]{2}{*}{ Sex } & \multirow{2}{*}{$\begin{array}{l}\text { Age } \\
\text { (years) }\end{array}$} & \multirow[t]{2}{*}{ Pain } & \multirow[t]{2}{*}{ History } & \multicolumn{2}{|c|}{ Routine MR protocol images } \\
\hline & & & & & Spinal abnormality & $\begin{array}{l}\text { Nerve root } \\
\text { impingement }\end{array}$ \\
\hline 1. Bilateral sacroiliitis & M & 20 & Low back pain & Irrelevant & - & - \\
\hline 2. Bilateral sacroiliitis & M & 19 & Low back pain & Irrelevant & - & - \\
\hline 3. Bilateral sacroiliitis & M & 24 & Low back pain & Irrelevant & $\begin{array}{l}\text { Lumbar } \\
\text { spondylosis }\end{array}$ & - \\
\hline 4. Bilateral sacroiliitis & $\mathrm{F}$ & 24 & $\begin{array}{l}\text { Bilateral } \\
\text { sciatica }\end{array}$ & Irrelevant & - & - \\
\hline 5. Stress fracture of right sacral alum & M & 39 & $\begin{array}{l}\text { Bilateral } \\
\text { sciatica }\end{array}$ & Irrelevant & Disc herniation & Positive \\
\hline 6. Stress fracture of right sacral alum & M & 30 & Right sciatica & Irrelevant & - & - \\
\hline 7. Stress fracture of proximal left femur & M & 36 & Low back pain & Irrelevant & Spondylolisthesis & Positive \\
\hline 8. Stress fracture of proximal right femur & M & 60 & Right sciatica & Irrelevant & $\begin{array}{l}\text { Lumbar } \\
\text { spondylosis }\end{array}$ & - \\
\hline $\begin{array}{l}\text { 9. Occult inter-trochanteric fracture of left } \\
\text { femur }\end{array}$ & $\mathrm{F}$ & 25 & Left sciatica & Trauma & - & - \\
\hline 10. Occult pelvic fracture & $\mathrm{F}$ & 20 & Left sciatica & Trauma & - & - \\
\hline 11. Left Iliac wing metastasis & M & 45 & $\begin{array}{l}\text { Bilateral } \\
\text { sciatica }\end{array}$ & $\begin{array}{l}\text { Vertebral } \\
\text { metastasis }\end{array}$ & Vertebral metastasis & Positive \\
\hline 12. Right Iliac wing metastasis & $\mathrm{F}$ & 22 & Right sciatica & NET of pancreas & - & - \\
\hline 13. Left TOH & $\mathrm{F}$ & 42 & Left sciatica & Irrelevant & $\begin{array}{l}\text { Lumbar } \\
\text { spondylosis }\end{array}$ & - \\
\hline 14. Bilateral Tarlov cysts & $\mathrm{F}$ & 32 & $\begin{array}{l}\text { Bilateral } \\
\text { sciatica }\end{array}$ & Irrelevant & - & - \\
\hline 15. Left gluteal muscular tendinopathy & $\mathrm{F}$ & 52 & Left sciatica & Irrelevant & $\begin{array}{l}\text { Lumbar } \\
\text { spondylosis }\end{array}$ & - \\
\hline
\end{tabular}

(NET neuroectodermal tumor)

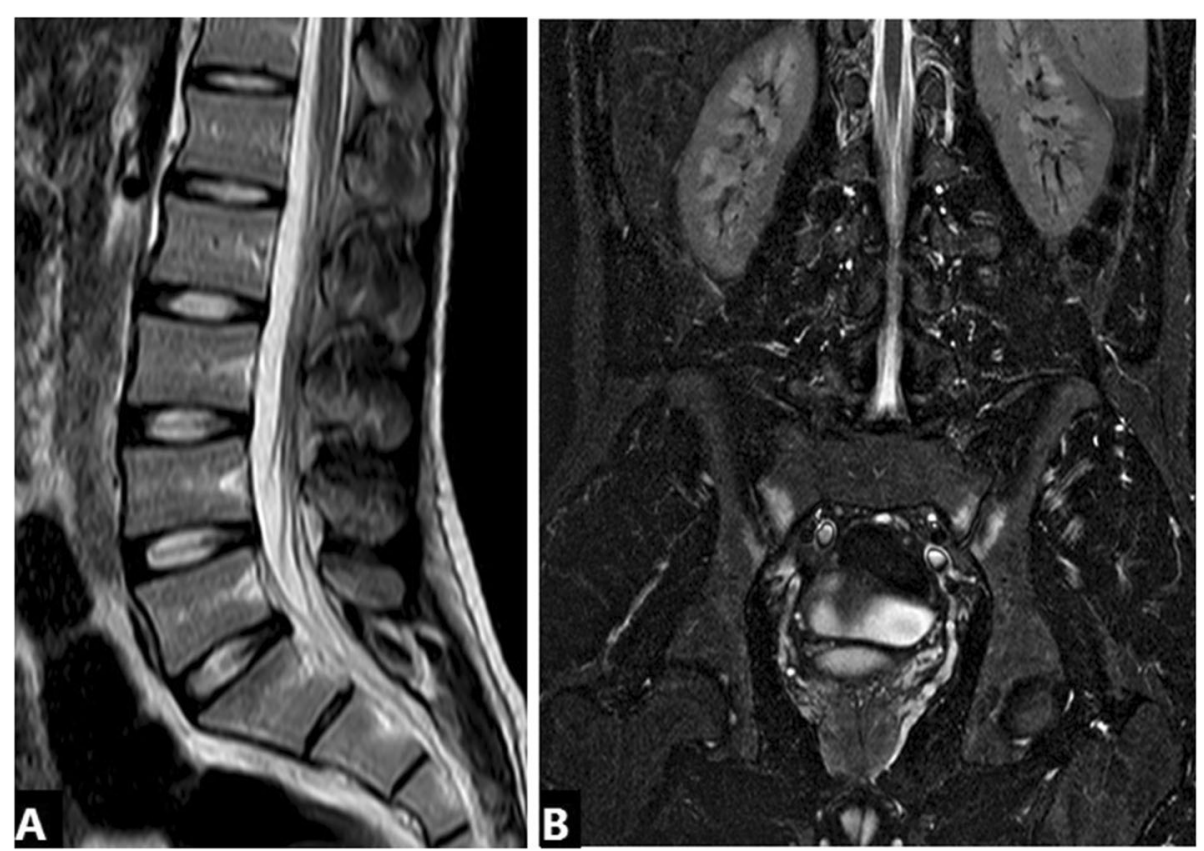

Fig. 2 A 24-year- old male patient, presenting with low back pain. a Routine MR protocol images are normal. b Additional coronal STIR images revealed subchondral edema in the inferior aspects of both sacroiliac joints denoting bilateral sacroiliitis 


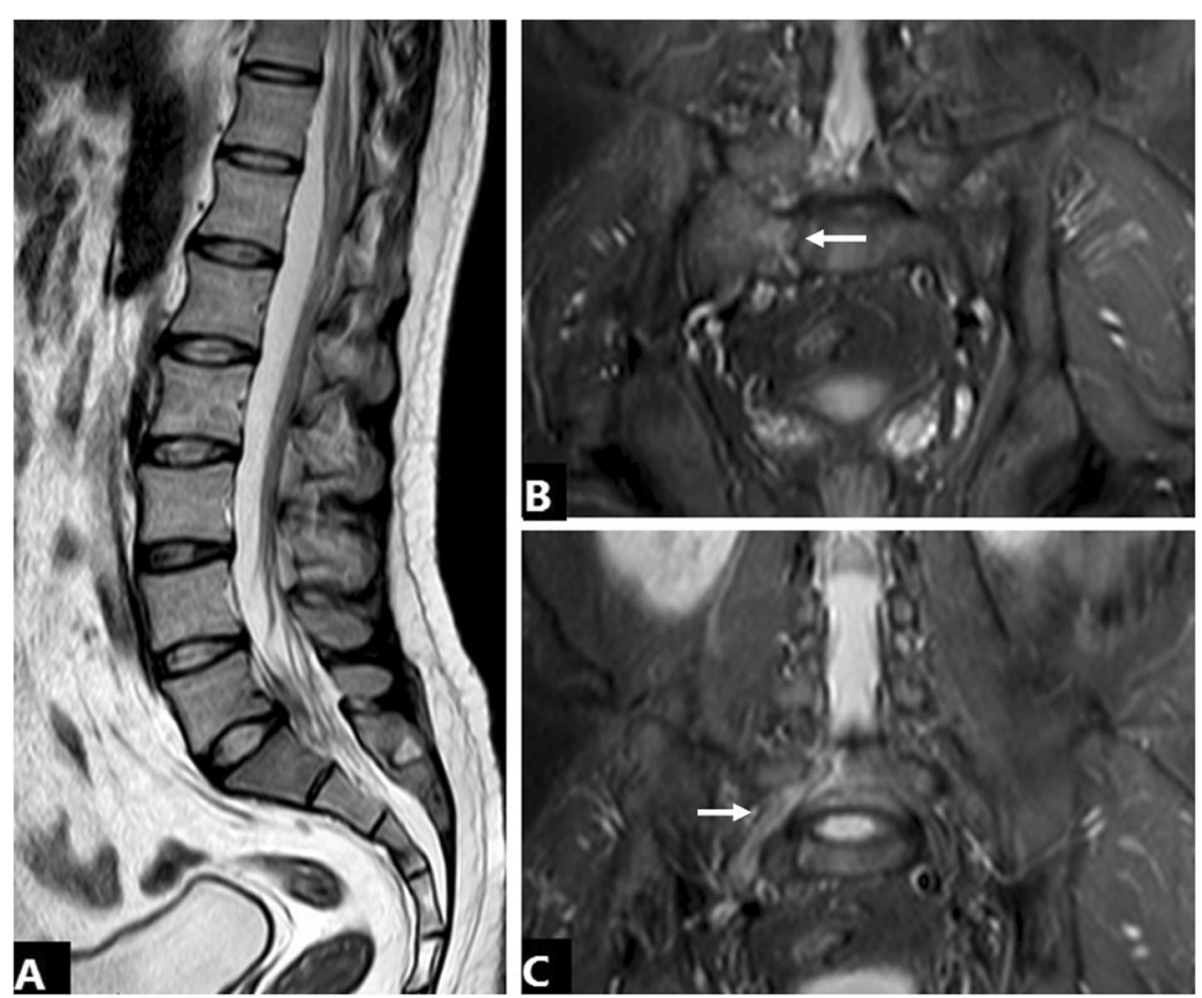

Fig. 3 A 30-year-old male patient, presenting with right sciatica. a Routine MR protocol images revealed mild thickening of the cauda equina nerve roots opposite S1 segment. b, c Additional coronal STIR images revealed marrow edema and a stress fracture at the right sacral alum (arrow in $\mathbf{b}$ ) and thick edematous right $\mathrm{S1}$ nerve root (arrow in $\mathbf{c}$ )

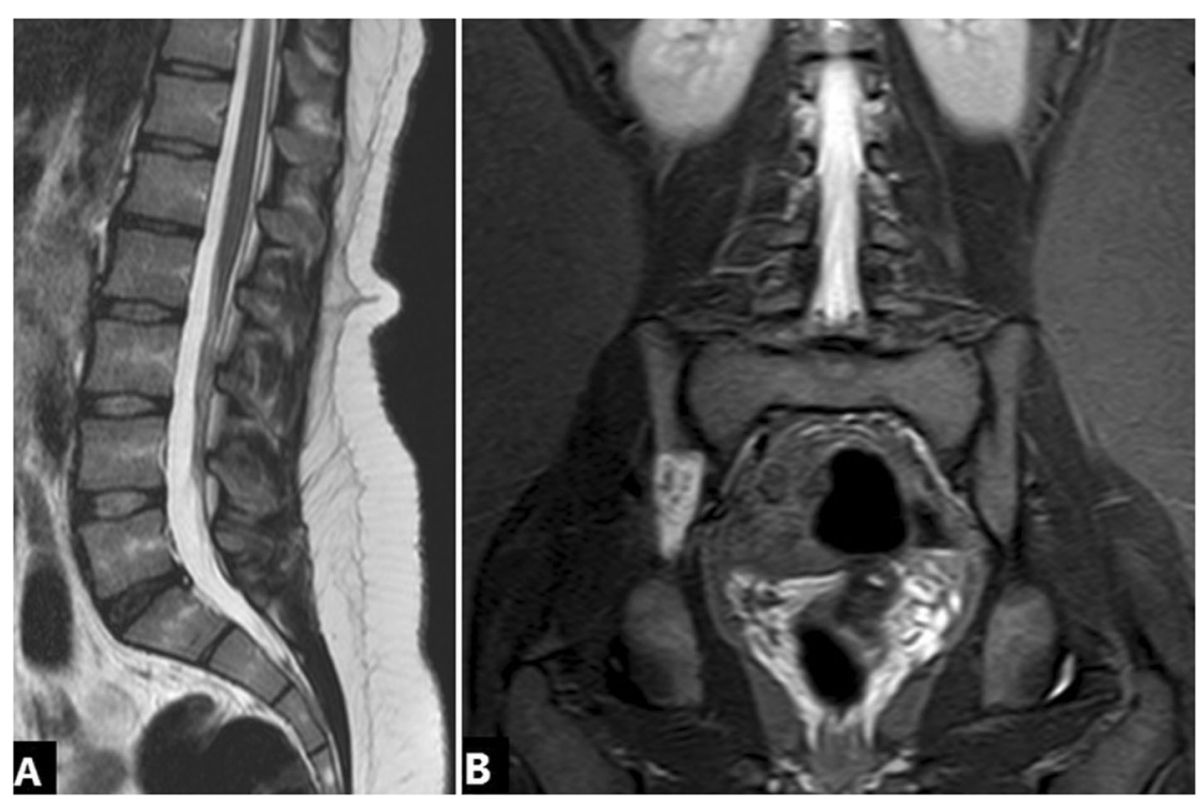

Fig. 4 A 22-year-old female patient, presenting with right sciatica. The patient is known to have pancreatic neuroendocrine tumor. a Routine MR protocol images revealed mild spondylodegenerative changes and L5/S1 tiny disc protrusion. No signs of nerve root impingement on other images. b Additional coronal STIR images revealed right iliac bone lesion. Bone scan revealed metastatic bone deposits 

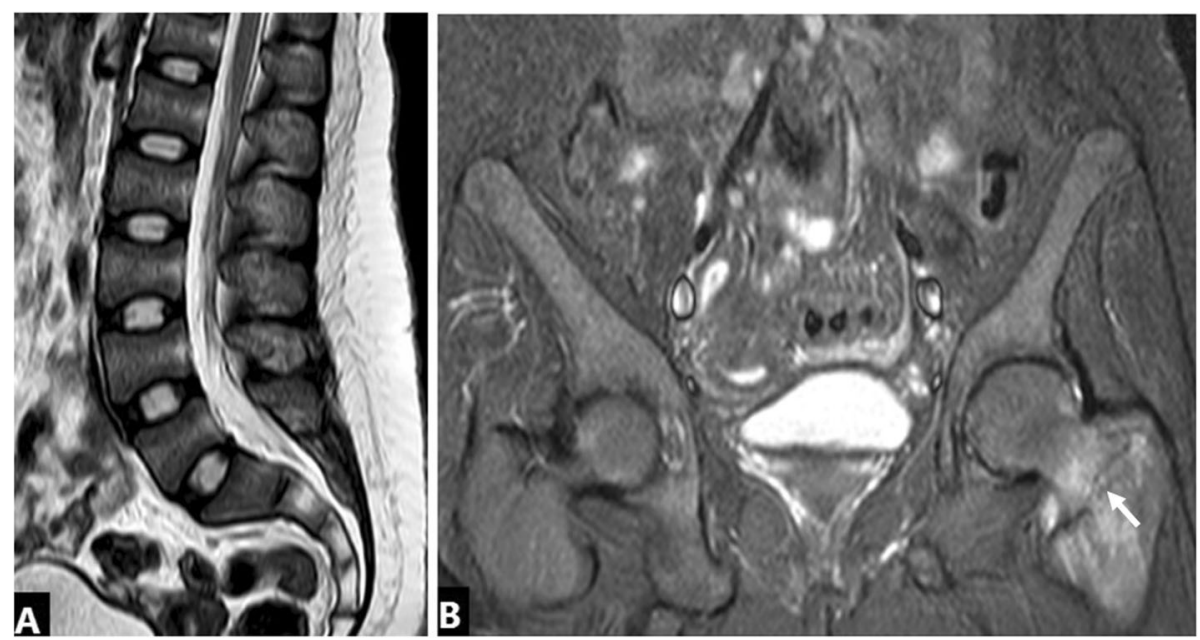

Fig. 5 A 25-year-old female patient, presenting with left sciatica. The patient gave history of recent trauma but plain radiography was normal on day of imaging. a Routine MR protocol images are normal. b Additional coronal STIR images revealed marrow edema at the left femoral neck and inter-trochanteric region, with a linear obliquely oriented impacted inter-trochanteric fracture line (arrow)

correlated with absence of spinal abnormalities and absence of nerve root impingement $(P$ value $=0.004$ and 0.002 respectively) on the routine MR protocol images (Table 3).

\section{Discussion}

The spectrum of radiating and non-radiating low back pain is a common annoying clinical problem. The majority of cases are attributed to discogenic lumbar nerve root compression, for which MRI is primarily used for evaluation [8-10]. It is not uncommon to encounter normal routine protocol MRI while a patient is still suffering from pain. Literature includes reports for many cases whose pain were attributed to sacro-iliac, gynecological, and other pelvic pathologies $[2-4,6,11-13]$. Others described nerve entrapment syndromes by muscles and tendons, as pyriformis syndrome [14-16].

In MR imaging, STIR is recognized as a beneficial sequence of high sensitivity in detection of bone as well as soft tissue pathologies through the illumination of high

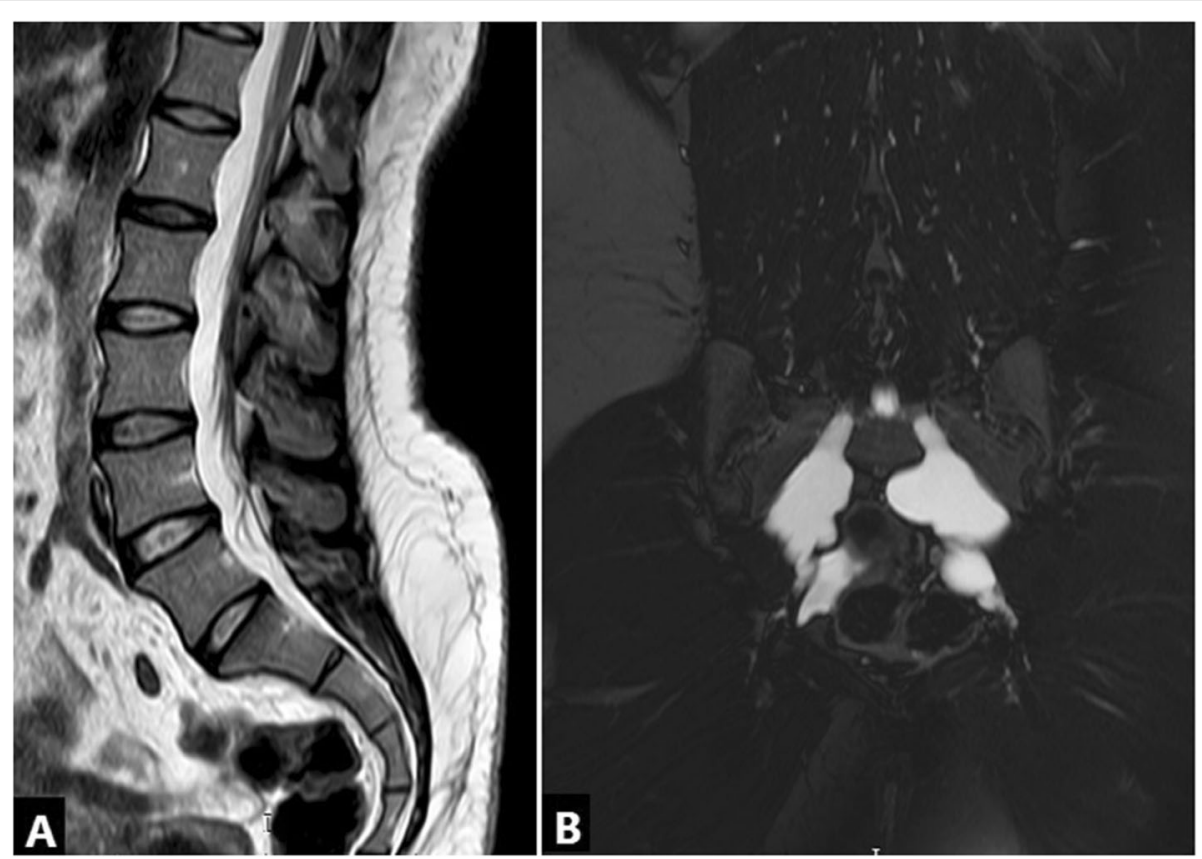

Fig. 6 A 32-year-old female patient, presenting with bilateral sciatica. a Routine MR protocol images are normal. b Additional coronal STIR images revealed bilateral large perineural cysts (Tarlov cysts) along the course of the sacral nerve roots associated with bone scalloping 
Table 3 Correlation between extra-spinal abnormalities detected on coronal STIR images and patients' sex, history of trauma, history of neoplasia, positive spinal abnormality, and signs of impingement on routine MR protocol images

\begin{tabular}{|c|c|c|c|c|c|c|}
\hline & \multirow[t]{2}{*}{ M } & \multirow[t]{2}{*}{$\mathrm{F}$} & \multirow[t]{2}{*}{ Trauma } & \multirow[t]{2}{*}{ Neoplasia } & \multicolumn{2}{|c|}{ Routine MR protocol images } \\
\hline & & & & & Spinal abnormality & Nerve root impingement \\
\hline Total no. of patients & 84 & 133 & 5 & 3 & 172 & 124 \\
\hline Extra-spinal abnormalities & 8 & 7 & 2 & 2 & 7 & 3 \\
\hline$P$ value & 0.228 & & 0.04 & 0.013 & 0.004 & 0.002 \\
\hline
\end{tabular}

water content pathologies against suppressed background fat signal. It allows homogeneous fat suppression, insensitive to field heterogeneity and applicable in low as well as high magnetic strength scanners [17-20].

The MR neurography imaging of lumbosacral plexus has also utilized STIR besides T1 sequences in axial and coronal planes; and the revolutionized MR neurography techniques in the era of 3-Tesla MRI scanners introduced high-resolution images of the nerve plexus through three dimensional (3D) isotropic acquisition imaging with the advantage of maximum intensity projection (MIP) as well as curvilinear reconstructed images. High-resolution images allow detection of signal changes along the nerves and small neuromas and may help to point to sites of entrapment [21-25]. While MR neurography provides visual analysis of the nerves, diffusion-weighted imaging can provide further quantitative assessment through measurement of the apparent diffusion coefficient value (ADC) and fraction anisotropy (FA) of entrapped nerves and nerve roots and using ADC values for differentiating benign from malignant neurogenic tumors [26-30]. Diffusion tensor imaging (DTI) and tractography can also be implemented to trace the neural tracts [31].

However, a standard coronal STIR sequence, covering the para-spinal and pelvic region, can be looked at as a primary problem solving method through detection of gross abnormalities overlooked on routine MRI protocol in extra-spinal sciatica. It is less demanding technically and available through scanners of different magnet strength making it a simple adjustment to routine imaging protocol.

In the current study, routine MR protocol images resulted in categorization of $21 \%$ of the included patients as normal while after adding coronal STIR images, $13 \%$ were categorized as normal; a discrepancy explained by the fact that 44 patients had extra-spinal abnormalities that were overlooked using the routine MR protocol images alone while detected on the additional coronal STIR images. A wide range of extra-spinal abnormalities were detected. Gynecological abnormalities (ovarian cysts and uterine fibroids) constituted half of these patients; however, the relation to the patients' presenting symptoms was considered equivocal. In 15 patients (6.9\%), we were confident that the extra-spinal abnormalities could explain pain and influenced the treatment plan, with absent nerve root impingement on routine protocol images in 12 of them.

In a study by Gleeson and his colleagues [7], additional coronal STIR images altered the diagnosis in $2.4 \%$ of the studied patients; which is much less than the reported percentage in our study. This can be explained that coronal images field of view in their study was limited to the sacrum and sacroiliac joints; while in our study, the FOV was extended down to the lesser trochanters of the femora allowing the detection of abnormalities within the pelvis and hips. In other studies, reported a percentage close to that in our study, $6.8 \%$ by Gupta and his colleagues and $5.7 \%$ by Laporte and his colleagues $[4,6]$.

Statistical analysis in our study aimed to identify the factors to which coronal STIR sequence has additional value and relevant application. It yielded significant correlation between the presence of extra-spinal cause of sciatica and positive history of trauma and neoplasm, normal routine protocol images, and absent nerve root impingement on routine protocol images. Extra-spinal sciatica was more prevalent in the age groups ranging from 20 to 39 years, being of significance in the age group: 20-29 years. In their study, Laporte et al. reported significant association between extra-spinal cause of pain and absent disco-radicular impingement on routine images [4].

The main limitation of the study is that we could not follow the patients diagnosed having gynecological abnormalities to correlate the presence of such abnormalities to the patients' pain, worth to mention that they constituted half of the detected extra-spinal abnormalities and have been reported in various case reports as unusual causes of sciatica. Yet additional coronal STIR images could detect the presence of gynecological and other pelvic abnormalities which may be referred to for consideration by the clinician in otherwise normal routine images protocol.

\section{Conclusion}

Additional coronal STIR images with FOV extending from the mid abdomen down to the femoral lesser trochanters identify extra-spinal abnormalities that are overlooked on routine MRI protocol images in patients 
presenting with sciatica and sciatica-like symptoms. Extra-spinal causes of sciatica are more prevalent in young adults. There is a significant correlation between extra-spinal sciatica and positive history of trauma and neoplasm, normal, or absent signs of nerve root impingement on routine protocol images.

\section{Abbreviations}

3D: 3 Dimensions; ADC: Attenuation diffusion coefficient; DICOM: Digital Imaging and Communications in Medicine; DTI: Diffusion tensor imaging; FA: Fraction anisotropy; FOV: Field of view; IR: Inversion recovery; MIP: Maximum intensity projection; MR: Magnetic resonance; MRI: Magnetic resonance imaging; SPSS: Statistical Package for the Social Sciences; STIR: Short TI inversion recovery; TE: Echo time; TR: Repetition time

\section{Acknowledgements}

The authors acknowledge Dr. Manar Hussein for her valuable contribution to this work.

\section{Authors' contributions}

RZ and MS worked on the conception, analysis, and interpretation of data. MS had major contribution to writing the manuscript. RZ revised the drafts and made the necessary editing and adjustments to the final approved version. Both authors read and approved the final manuscript.

\section{Funding}

There are no sources of funding to be declared.

\section{Availability of data and materials}

The datasets used and/or analyzed during the current study are available from the corresponding author on reasonable request.

\section{Ethics approval and consent to participate}

The study has been approved by the "Ethical Committee of Faculty of Medicine, Cairo University", in compliance with Helsinki Declaration. A written consent was obtained from the included patients. This study was not issued a reference number by the ethical committee because back then, the approving committee did not apply issuing serial numbers.

\section{Consent for publication}

All the included patients signed an informed consent to publish the data contained within this study.

\section{Competing interests}

The authors declare that they have no competing interests.

Received: 28 November 2019 Accepted: 2 December 2019

Published online: 10 December 2019

\section{References}

1. Valat J-P, Genevay S, Marty M et al (2010) Sciatica. Best Pract Res Clin Rheumatol 24:241-252. https://doi.org/10.1016/j.berh.2009.11.005

2. Yoshimoto M, Kawaguchi S, Takebayashi T et al (2009) Diagnostic features of sciatica without lumbar nerve root compression. J Spinal Disord Tech 22: 328-333. https://doi.org/10.1097/BSD.0b013e31817dc46d

3. Ergun T, Lakadamyali $H$ (2010) CT and MRI in the evaluation of extraspinal sciatica. Br J Radiol 83:791-803. https://doi.org/10.1259/bjr/76002141

4. Laporte C, Albert J-D, Duvauferrier R et al (2014) MRI investigation of radiating pain in the lower limbs: value of an additional sequence dedicated to the lumbosacral plexus and pelvic girdle. Am J Roentgenol 203:1280-1285. https://doi.org/10.2214/AJR.13.11884

5. Brant WE, Helms CA (2012) Fundamentals of diagnostic radiology. Wolters Kluwer Health/Lippincott Williams \& Wilkins, Philadelphia

6. Gupta R, Mittal P, Mittal A et al (2015) Additional merit of coronal STIR imaging for MR imaging of lumbar spine. J Craniovertebral Junction Spine 6:12. https://doi.org/10.4103/0974-8237.151582

7. Gleeson TG, O'Connell MJ, Duke D et al (2005) Coronal oblique turbo STIR imaging of the sacrum and sacroiliac joints at routine MR imaging of the lumbar spine. Emerg Radiol 12:38-43. https://doi.org/10.1007/ s10140-005-0440-1

8. Wassenaar M, van Rijn RM, van Tulder MW et al (2012) Magnetic resonance imaging for diagnosing lumbar spinal pathology in adult patients with low back pain or sciatica: a diagnostic systematic review. Eur Spine J 21:220-227. https://doi.org/10.1007/s00586-011-2019-8

9. Tawa N, Rhoda A, Diener I (2016) Accuracy of magnetic resonance imaging in detecting lumbo-sacral nerve root compromise: a systematic literature review. BMC Musculoskelet Disord 17. https://doi.org/10.1186/ s12891-016-1236-z

10. Dullerud R (1999) Radiological imaging in lumbago and sciatica. Tidsskr Den Nor Laegeforening Tidsskr Prakt Med Ny Raekke 119:1778-1781

11. Al-Khodairy AT, Gerber BE, Praz G (1995) Adenomyosis--an unusual cause of sciatic pain. Eur Spine J 4:317-319

12. Cottier JP, Descamps $P$, Sonier CB, Rosset $P$ (1995) Sciatic endometriosis: MR evaluation. AJNR Am J Neuroradiol 16:1399-1401

13. Voyadzis JM, Bhargava P, Henderson FC (2001) Tarlov cysts: a study of 10 cases with review of the literature. J Neurosurg 95:25-32

14. Filler AG, Haynes J, Jordan SE et al (2005) Sciatica of nondisc origin and piriformis syndrome: diagnosis by magnetic resonance neurography and interventional magnetic resonance imaging with outcome study of resulting treatment. J Neurosurg Spine 2:99-115. https://doi.org/10.3171/ spi.2005.2.2.0099

15. Murata Y, Ogata S, Ikeda Y, Yamagata M (2009) An unusual cause of sciatic pain as a result of the dynamic motion of the obturator internus muscle. Spine J 9:e16-e18. https://doi.org/10.1016/j.spinee.2009.01.004

16. Kosukegawa I, Yoshimoto M, Isogai S et al (2006) Piriformis syndrome resulting from a rare anatomic variation. Spine 31:E664-E666. https://doi. org/10.1097/01.brs.0000231877.34800.71

17. Tokuda O, Harada Y, Matsunaga N (2009) MRI of soft-tissue tumors: fast STIR sequence as substitute for T1-weighted fat-suppressed contrast-enhanced spin-echo sequence. Am J Roentgenol 193:1607-1614. https://doi.org/10. 2214/AJR.09.2675

18. Lakadamyali H, Tarhan NC, Ergun T et al (2008) STIR sequence for depiction of degenerative changes in posterior stabilizing elements in patients with lower back pain. Am J Roentgenol 191:973-979. https:// doi.org/10.2214/AJR.07.2829

19. Del Grande F, Santini F, Herzka DA et al (2014) Fat-suppression techniques for 3-T MR imaging of the musculoskeletal system. Radiographics 34:217233. https://doi.org/10.1148/rg.341135130

20. Vanel D (2004) MRI of bone metastases: the choice of the sequence. Cancer Imaging 4:30-35. https://doi.org/10.1102/1470-7330.2003.0029

21. Cho Sims G, Boothe E, Joodi R, Chhabra A (2016) 3D MR neurography of the lumbosacral plexus: obtaining optimal images for selective longitudinal nerve depiction. Am J Neuroradiol 37:2158-2162. https:// doi.org/10.3174/ajnr.A4879

22. Lewis AM, Layzer R, Engstrom JW et al (2006) Magnetic resonance neurography in extraspinal sciatica. Arch Neurol 63:1469. https://doi.org/10. 1001/archneur.63.10.1469

23. Ailianou A, Fitsiori A, Syrogiannopoulou A et al (2012) Review of the principal extra spinal pathologies causing sciatica and new MRI approaches. Br J Radiol 85:672-681. https://doi.org/10.1259/bjr/84443179

24. Moore KR, Tsuruda JS, Dailey AT (2001) The value of MR neurography for evaluating extraspinal neuropathic leg pain: a pictorial essay. AJNR Am J Neuroradiol 22:786-794

25. Muniz Neto FJ, Kihara Filho EN, Miranda FC et al (2018) Demystifying MR neurography of the lumbosacral plexus: from protocols to pathologies. Biomed Res Int 2018:1-20. https://doi.org/10.1155/2018/9608947

26. Wada K, Hashimoto T, Miyagi R et al (2017) Diffusion tensor imaging and tractography of the sciatic nerve: assessment of fractional anisotropy and apparent diffusion coefficient values relative to the piriformis muscle, a preliminary study. Skelet Radiol 46:309-314. https:// doi.org/10.1007/s00256-016-2557-6

27. Eguchi Y, Ohtori S, Orita S et al (2011) Quantitative evaluation and visualization of lumbar foraminal nerve root entrapment by using diffusion tensor imaging: preliminary results. Am J Neuroradiol 32:1824-1829. https:// doi.org/10.3174/ajnr.A2681

28. Eguchi Y, Ohtori S, Yamashita M et al (2011) Diffusion-weighted magnetic resonance imaging of symptomatic nerve root of patients with lumbar disk herniation. Neuroradiology 53:633-641. https://doi.org/ 10.1007/s00234-010-0801-7 
29. Eguchi Y, Ohtori S, Yamashita M et al (2010) Clinical applications of diffusion magnetic resonance imaging of the lumbar foraminal nerve root entrapment. Eur Spine J 19:1874-1882. https://doi.org/10.1007/ s00586-010-1520-9

30. Razek AAKA, Ashmalla GA (2018) Assessment of paraspinal neurogenic tumors with diffusion-weighted MR imaging. Eur Spine J 27:841-846. https://doi.org/10.1007/s00586-017-5265-6

31. Wako $Y$, Nakamura J, Eguchi $Y$ et al (2017) Diffusion tensor imaging and tractography of the sciatic and femoral nerves in healthy volunteers at 3T. J Orthop Surg 12. https://doi.org/10.1186/s13018-017-0690-0

\section{Publisher's Note}

Springer Nature remains neutral with regard to jurisdictional claims in published maps and institutional affiliations.

\section{Submit your manuscript to a SpringerOpen ${ }^{\circ}$ journal and benefit from:}

- Convenient online submission

- Rigorous peer review

- Open access: articles freely available online

High visibility within the field

- Retaining the copyright to your article

Submit your next manuscript at $\boldsymbol{\nabla}$ springeropen.com 